\title{
Vaccination with Single Plasmid DNA Encoding \\ IL-12 and Antigens of Severe Fever with Thrombocytopenia Syndrome Virus Elicits Complete Protection in IFNAR Knockout Mice ${ }^{\dagger}$
}

\author{
Nam-Hyuk Cho \\ College of Medicine, Seoul National University, Seoul 03080, Korea; chonh@snu.ac.kr \\ + Presented at Viruses 2020-Novel Concepts in Virology, Barcelona, Spain, 5-7 February 2020. \\ Published: 24 June 2020
}

\begin{abstract}
Severe fever with thrombocytopenia syndrome (SFTS) is an emerging tick-borne disease caused by SFTS virus (SFTSV) infection. Despite the gradual increase in SFTS cases and high mortality in the endemic region, no specific viral therapy nor vaccine is available. Here, we developed single recombinant plasmid DNA encoding SFTSV genes Gn and Gc together with the NP-NS fusion antigen as a vaccine candidate. The viral antigens were fused with Fms-like tyrosine kinase-3 ligand (Flt3L) and the IL-12 gene was incorporated into the plasmid to enhance cellmediated immunity. Vaccination with the DNA can provide complete protection of IFNAR KO mice upon lethal SFTSV challenge, whereas immunization with a plasmid lacking the IL-12 gene resulted in partial protection. Since we failed to detect antibodies against the surface glycoproteins $\mathrm{Gn}$ and Gc in the immunized mice, antigen-specific cellular immunity, as confirmed by enhanced antigenspecific $\mathrm{T}$ cell responses, might play a major role in protection. Finally, we evaluated the degree of protective immunity after protein immunization that was provided by individual glycoproteins $\mathrm{Gn}$ or Gc. Although both protein antigens induced significant levels of neutralizing activity against SFTSV, Gn vaccination resulted in relatively higher neutralizing activity and better protection than Gc vaccination. However, both antigens failed to provide complete protection. Given that the DNA vaccines have failed to induce sufficient immunogenicity in human trials when compared with protein vaccines, optimal combinations of DNA and protein vaccine, proper selection of target antigens, and incorporation of an efficient adjuvant need to be further investigated for SFTS vaccine development.
\end{abstract}

Keywords: severe fever with thrombocytopenia syndrome virus; DNA vaccine; protection; IFNAR KO mouse

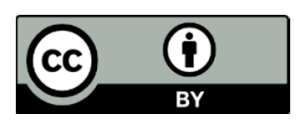

(C) 2020 by the authors. Licensee MDPI, Basel, Switzerland. This article is an open access article distributed under the terms and conditions of the Creative Commons Attribution (CC BY) license (http://creativecommons.org/licenses/by/4.0/). 